\title{
Algebra of Finite-Valued Functions: Classification of Functions and Subalgebras, Essential and Fictitious Subalgebras
}

\author{
Maydim Malkov \\ Department of mathematics, Russian Research Center for Artificial Intelligence, Moscow, Russia
}

\section{Email address:}

mamalkov@gmail.com

\section{To cite this article:}

Maydim Malkov. Algebra of Finite-Valued Functions: Classification of Functions and Subalgebras, Essential and Fictitious Subalgebras. Pure and Applied Mathematics Journal. Vol. 8, No. 2, 2019, pp. 30-36. doi: 10.11648/j.pamj.20190802.11

Received: April 28, 2019; Accepted: June 2, 2019; Published: June 26, 2019

\begin{abstract}
The classification of subalgebras of every algebra of finite-valued functions is constructed. Classes of this classifications do not intersect. Each class contains subalgebras with the same number of functions in minimal basis. Class with ordinal number 0 contains subalgebras that have no basis. The class with finite ordinal number $\mathrm{n}$ contains subalgebras whose minimal basis has $\mathrm{n}$ functions. The set of subalgebras of this class are countable. There is a class with infinite ordinal number. Subalgebras of this class have a minimal basis with infinite number of functions. The set of these subalgebras is continual. Only the class with ordinal number 1 is essential, all other classes are fictitious, since they are useless to classify functions. But classification of functions is the main problem of the algebra of finite-valued functions. A class of this classification is a set of functions extracted from one-member bases of a subalgebra. Each function generates by superpositions some subalgebra, and only this subalgebra. So, this function belongs to only one class. All these classes of functions belong to the class 1 of subalgebras. All subalgebras from classes with the other ordinal numbers are useless to classify functions. The set of these fictitious subalgebras is continual, the set of essential subalgebras are countable. The top level of the classification of functions contains the algebra of finite-valued functions. Next level contains maximal subalgebras. According to I.G. Rosenberg, there are 6 sets of maximal subalgebras. I.G. Rosenberg was wrong to state the set of his quasilinear functions be maximal. Only Yablonsky's set of quasilinear functions is maximal. The sixth Rosenberg's set also turns to be wrong. This right set was built by A.I. Maltsev. But from 6 sets only 3 sets contain essential subalgebras. And all maximal essential subalgebras containing 3-valued 2-place functions are built.
\end{abstract}

Keywords: Algebra of Finite-Valued Functions, Classification of Finite-Valued Functions, Post Algebras, Maximal Algebras

\section{Introduction}

The algebra of functions of value $k$ has a carrier to be the set of all functions of this value and has an operation set called superpositions (compositions). A mathematical definition of superpositions was given by A. I. Maltsev (see Section 3).

The algebra of many-valued functions (hereinafter, of $k>2$ ) are denoted by $\mathrm{P}_{\mathrm{k}}$ in honor of E. L. Post ${ }^{1}$. Subalgebras of algebra of functions of value $k$ have operations of superpositions and have carriers to be sets of functions closed

${ }^{1}$ A.I. Maltsev called Post algebra an algebra with superpositions and with functions of any value including continual. E.L. Post used such algebra for classification of two-valued functions. by superpositions.

Further algebra of functions of value $k$ are called briefly function algebra, subalgebras of the function algebra are called algebras, functions of value $k$ are called functions.

Maximal algebras of the function algebra are generally accepted to call algebras, which contained only in this algebra.

Maximal algebras attract special attention of mathematicians in connection with classification of functions. The classification is the main problem of the function algebra. The carriers of maximal algebras are the second level of the classification, the first level contains the class of all functions.

A complete classification of two-valued functions was given by E. L. Post $[1,2]$. The complete classification of three-valued functions is still not constructed because of an 
incomparably greater number of sets of functions closed by superpositions.

Many authors tried to solve the problem of classifying many-valued functions using stronger closure operations than superpositions.

One of the first was the work of A. V. Kuznetsov [3], in which a parametric closure was introduced. A more complete study of the closures is got by S. S. Marchenkov. These are the 1L-closure [4], S-closure [5, 6], equational closure [7, 8], and FE-closure [9, 10].

Better way is to use superpositions for maximal and pre-maximal algebras.

The finite number of maximal algebras was established by A. V. Kuznetsov [11]. An asymptotically exact formula for the number of maximal algebras was given in [12]. S. V. Yablonsky [13] established that the number of maximal algebras of three-valued functions is 18 . The number of pre-maximal algebras of three-valued functions was found by D. Lau [14]. It is equal to 158,5 of these pre-maximal algebras have a finite set of subalgebras, 7 have a countable set, and the others have a continual set. The number of maximal subalgebras for any $k$ was found by I. G. Rosenberg [15].

New results for the number of maximal algebras are given in this article. It turned out that there are fictitious (useless, see section 2) algebras. The set of these algebras is continual, and the set of essential (non-fictious) algebras is countable.

\subsection{Basic Definitions}

All algebras have the same set of operations and differ only by carriers. So, an algebra and its set of functions are commonly identified, i.e. one calls algebra a carrier of algebra. In particular, intersection of algebras is intersection of their carriers. An intersection of algebras is an algebra, a union of algebras may not be an algebra, but this union of algebras generates an algebra containing them (this union contains other algebras too).

This identification simplifies the formulation of mathematical statements.

Fictitious variables are variables useless for calculation of their function.

Fictitious function contains fictitious variables. It is generally accepted to equal all functions in which fictitious variables are added or removed. This definition is incorrect because these functions have different tables. But tables are sets of rows, and sets are equal if their elements are equal. In applied mathematics, the fictitious functions are removed, they are useless.

Complete functions and complete set of functions generate all functions.

Basis of an algebra is a set of functions generating all functions of this algebra, if any properly subsets of this set does not generate this algebra.

Minimal basis has minimal elements. An algebra can have several minimal bases. The definition with one minimal basis does not need below.

Diagonals are table rows for a function with equal values of variables.
Family of functions, briefly family, is a set of functions with diagonals $f(x, \ldots, x)=x$ for some $x$. The concept of families is a further extension of this concept in two-valued logic.

An algebra belongs to a family (is algebra of a family) if it has all bases to belong to this family. But an algebra of a family may contain algebras of other families.

Algebra is fictitious if it is useless for classification of functions (see section 2).

Essentially algebra is not fictitious.

Rosenberg set is one of 6 sets of maximal algebras used in Rosenberg classification of maximal algebras.

\subsection{Notation}

The letter $k$ is used for values of functions (in particular, the set of functions of values 0 is empty, the set of functions of values 1 contains only one function in each arity).

The set of all values is denoted by $\{0, \ldots, k-1\}$ (the common notation $E_{k}$ is not mnemonic, mnemonic, for example, $N_{k}$, that is a set of the first $k$ natural numbers, the number 0 is natural).

The letter $s$ denotes a one-ary function that takes all values and is used to construct dual functions.

The operations of addition, multiplication and equality use modulo $k$.

\section{Fictitious Algebras}

\subsection{Definitions}

Each theory has useless objects. It is generally accepted to call these objects fictitious (in particular, fictitious variables are useless to calculate their function). The theory of function algebra has no exception.

The main problem of this algebra is classification of functions. But there are fictitious algebras that are useless for classifying functions.

Indeed, each function generates some algebra. This function belongs to single (one-member) basis of this algebra. In the following, the functions of single bases are called generators.

The set of generators of algebra is a class of natural classification (i.e. classification with non-intersecting classes), since the function cannot belong to several classes of generators. Each function generates only one algebra and is contained in the class of generators of this algebra.

Algebras with single bases define the classification of functions, since such an algebra has a unique class of generators, and each function belongs to only one such class.

The other algebras are fictitious, since they are useless for classifications of functions. Their number is continual. The number of essential algebras is countable. This is natural, since in any theory, fictitious objects are incomparably more than essential objects. In particular, one essential function has an infinite number of fictitious functions obtained by adding fictitious variables to this essential function.

Therefore, the statement about the essential difference 
between algebras of two-valued and many-valued functions is erroneous [16]. This statement is based on the continuity of fictitious algebras. But fictitious objects are deleted (not used) in any theory.

\subsection{Classification of Algebras}

Classification of functions begins with the classification of their algebras. This classification contains all algebras. It allows to find fictitious algebras and to exclude them. Then classification only essential algebras is continued.

This classification is realized by number of members in minimal bases [17].

Such a classification is natural, since its classes are not intersected. Other existing classifications are usually not natural.

The algebra of two-valued functions contains only 3 classes of algebras. They have minimal bases from one to three functions [18]. The algebra of many-valued functions contains all classes with bases from zero to infinite number of functions [16]. A basis with zero number of functions belongs to algebras that have no basis. A basis with infinite number of functions belongs to algebras with infinite basis.

The class of algebras with infinite basis contains a continual set of them. The other classes with finite bases contain a countable set of subalgebras.

The class with a single basis contains only essential algebras. The remaining classes contain only fictitious algebras.

\section{Main Theorems. Family of Functions}

\subsection{Superpositions}

Proofs of theorems use superpositions.

A. I. Maltsev [19] called superpositions 4 operations of algebras of functions of any value ${ }^{2}$. These are operations:

a) cyclic permutation of variables, i.e. the first variable is placed behind all the variables; as a result, the second variable becomes the first;

b) permutation of the first and last variables;

c) identifies the first and last variables;

d) substitution of a function in the first variable of this or of any other function.

The first two operations allow to perform any variable permutation. Any variable permutation is commonly referred to as a permutation operation.

A. I. Maltsev gave a mathematically precise definition of these operations [19]. Some improvement is given in [20].

\subsection{Families of Functions}

Families of functions will be used to construct maximal algebras.

A family of functions preserves constant $c$ if for any

${ }^{2}$ Algebra with these four operations was used by E. L. Post. There are fictitious functions in this algebra. S.V. Yablonsky [13] used another algebra, with another operation that allowed removing all fictitious functions. function $f$ the diagonal $f(c, \ldots, c)=c$ holds

Families of functions are a powerful means of classifying algebras, including maximal ones. All Post results in algebra of two-valued functions were obtained by him using families of functions.

E.L. Post built 4 families in the algebra of two-valued functions: $\alpha, \beta, \gamma$ and $\delta$. The functions of the $\alpha$ family preserve all the constants, the families of $\beta$ only constant 1 , the families of $\gamma$ only constant 0 . The functions of the $\delta$ family do not save anything.

In algebra of many-valued functions, the number of families is equal to the number of all possible combinations of constants, i.e. equals $2^{k}$. Functions of $\alpha$ family also preserve all constants, the functions of the $\delta$ family also do not save anything. Other designations of families are not copied from the algebra of two-valued functions.

Families of functions do not intersect, each function belongs to only one family, but the function of this family can generate functions of another family.

In the following, families of functions are called briefly families and are denoted by sets of preserved constants. In particular, the family $\delta$ is denoted by the empty set \{\} , the family $\alpha$ is denoted by the set of all constants $\{0, \ldots, k-1\}$. Families with one constant are called constant families. Algebras generated by functions of a family have name of this family. But these algebras can contain algebras of other families. Algebras containing functions of constant families are called constant algebras. Only constant algebras and algebras $\delta$ can be maximal. The other algebras are contained in constant algebras.

Families have the following properties.

Theorem 1. The family function $X=\left\{j_{1}, \ldots, j_{m}\right\}$ can generate functions of family $Y \supseteq X$ and cannot generate functions of family $Y \subset X$.

Proof. Variables permutation and identification do not change a family of functions. A function substitution can change a family.

Let $f\left(x_{1}, \ldots, x_{n}\right)$ belong to the family $X$ and let

$$
f_{0}\left(x_{1}, \ldots, x_{2 n-1}\right)=f\left(f\left(x_{1}, \ldots, x_{n}\right), x_{n+1}, \ldots, x_{2 n-1}\right)
$$

Let the function $f_{0}$ belong to the family $Y$, and we need to prove that $Y \supseteq X$. Then $f_{0}(j, \ldots, j)=j$ for any $j \in X$. In this case, $j \in Y$ for all $j \in X$. Let $j \notin X$. Then $f(j, \ldots, j)=c \neq$ $j$, but there is $f$ ( $f$ is an arbitrary function) such that $f(c, j, \ldots, j)=j$. Therefore, $Y \supset X$. And there is $f$ such that $f(c, j, \ldots, j) \neq j$ for any $j \notin X$. Therefore, $Y=X$. As a result, $Y \subseteq X$

Corollary 1. In algebra of many-valued functions, as in algebra of two-valued functions, the function of $\alpha$ family generates functions of this family only. The family function $\delta$ can generate functions of any family.

Proof. The family $\alpha$ has the notation $X=\{0, \ldots, k-1\}$, and $X$ cannot be a proper subset of any other set in a $k$ -valued algebra. The family $\delta$ is denoted $X=\{\}$, and $X$ is a proper subset of any other set in the $k$-valued algebra.

Complete functions (usually called Sheffer functions) can belong only to family $\delta$.

The following theorem is devoted to the main property of 
classes of generators generating an essential algebra.

Theorem 2. If a function of a class of generators belongs to a certain family, then all functions of the class of generators belong to this family.

Proof. If a function of a class of generators belongs to family $\alpha$, then all functions of the class belong to this family, since the function of family $\alpha$ generates functions only of this family. If a function of class $X$ belongs to the family $\delta$, then all functions of this class must generate this $\delta$ function. By the previous theorem, only the $\delta$ function can generate the $\delta$ function.

The evidence for other families is similar.

An algebra is said to belong to a family, if this algebra has class of generators of this family. But this algebra may contain algebras of other families.

An algebra is said to belong to $\delta$ family, if its class of generators contains non-complete $\delta$ functions. An algebra is called algebra of complete $\delta$ functions, if its class of generators contains only complete $\delta$ functions.

Algebras of $\delta$ families have the following properties.

One function generates a function of $\delta$ family only if it is $\delta$ function, but two or more functions can generate $\delta$ function, even if they are not $\delta$ function.

Theorem 3. In the algebra of many-valued functions, as in the algebra of two-valued functions, two functions of families other than $\delta$ can generate a function of family $\delta$.

Proof. Let a function $f_{1}$ belong to family $X_{1}=$ $\left\{a_{1}, \ldots, a_{m_{1}}\right\}$, a function $f_{2}$ belongs to family $X_{2}=$ $\left\{b_{1}, \ldots, b_{m_{2}}\right\}$ and let $X_{1} \cap X_{2}=\varnothing$. Then the substitution of $f_{2}$ in $f_{1}$ has diagonals $f_{1}\left(f_{2}\left(a_{i}, \ldots, a_{i}\right), a_{i}, \ldots, a_{i}\right) \neq a_{i}$, since $f_{2}\left(a_{i}, \ldots, a_{i}\right)=c \neq a_{i}$, and there is $f_{1}\left(c, a_{i}, \ldots, a_{i}\right) \neq$ $a_{i}$ for all $a_{i}$. Therefore, $f_{1}$ and $f_{2}$ generate $\delta$ function.

Corollary 2. Two functions of a basis of essential algebra do not generate $\delta$ function if both functions are not $\delta$ functions.

Proof. The proved theorem is satisfiable only if $X_{1} \cap X_{2}=$ $\emptyset$. But in this case, $X_{1} \cap X_{2} \neq \varnothing$, since $X_{1}=X_{2}$ by the previous theorem. Both functions generate the same algebra. This algebra does not contain $\delta$ functions.

But two functions of basis of fictitious algebra can generate $\delta$ function. These two functions should belong to different families.

The following property of $\delta$ algebras belongs to maximal algebras.

Theorem 4. All essentially maximal algebras belong to constant families or $\delta$ families, i.e. do not belong to $\alpha$ family.

Proof. Families $\alpha$ functions are intersection of families of functions that preserve one constant.

In the algebra of two-valued functions, only one maximal algebra belongs to the family $\delta$, it is a self-dual algebra. In the algebras of many-valued functions, the family $\delta$ contains several maximal algebras.

The following lemma is used to construct maximal algebras.

Lemma 1 . The essentially maximal algebra is not a union of algebras.

Proof. The union of algebras is fictitious, since its basis contains the bases of these algebras.

\section{Maximal Algebras}

\subsection{Algebra of Two-Valued Functions}

5 algebras are considered maximal: $C_{2}, C_{3}, D_{3}, A_{1}, L_{1}$. Only constant and $\delta$ algebras can be essentially maximal, i.e. $C_{2}, C_{3}, D_{3}$.

The algebra $D_{3}$ is essential, its minimal basis is a function $x_{1} \bar{x}_{2} \vee x_{1} \bar{x}_{3} \vee \bar{x}_{2} \bar{x}_{3}[21]$.

E.L. Post considered that the algebras $C_{2}, C_{3}$ do not have single bases. I.e. these algebras are fictitious.

It suffices to prove that the algebra $C_{3}$ is essential. By duality principle, the algebra $C_{2}$ will be essential too.

The algebra $C_{3}$ has a basis $x_{1} \bar{x}_{2} \bar{x}_{3} \vee \bar{x}_{1} x_{2} x_{3}$.

Indeed, let $x_{2}=x_{3}$ in $x_{1} \bar{x}_{2} \bar{x}_{3} \vee \bar{x}_{1} x_{2} x_{3}$. Then the function $x_{1} \bar{x}_{2} \vee \bar{x}_{1} x_{2}=x_{1}+x_{2}$ is generated.

Substitute the function $x_{1} \bar{x}_{2} \bar{x}_{3} \vee \bar{x}_{1} x_{2} x_{3}$ instead of the first variable of this function: $\left(x_{1} \bar{x}_{2} \bar{x}_{3} \vee \bar{x}_{1} x_{2} x_{3}\right) \bar{x}_{4} \bar{x}_{5} \mathrm{~V}$

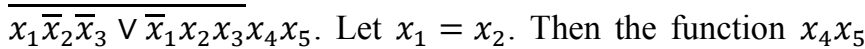
is generated.

It is known [21] that both generated functions form a basis of the algebra $C_{3}$. Therefore, the function $x_{1} \bar{x}_{2} \bar{x}_{3} \vee \bar{x}_{1} x_{2} x_{3}$ is also a basis.

Further we study algebras of many-valued functions.

\subsection{Monotone Algebras}

All monotone algebras with different order are isomorphic. In particular, there is one-to-one correspondence of inclusion diagrams of algebras with a natural order and a different order, since the result of superpositions does not change with change of order. Superpositions only preserve order.

Consequently, the natural order is enough.

One of Hasse diagrams defines a linear order, the other diagrams define a partial order for $k>3$ (for $k=3$ there is only one Hasse diagram).

Lemma 2. Monotone $\delta$ functions do not exist.

Proof. Suppose that a monotone function $f$ is a $\delta$ function. Let $0<j_{1}<\ldots<j_{m}<k-1$ be a partial order (if $j_{i}=i \wedge$ $m=k-2$ then this order is usual). Then $f(0, \ldots, 0) \in$ $\left\{j_{1}, \ldots, j_{m}, k-1\right\}, f\left(j_{1}, \ldots, j_{1}\right) \in\left\{j_{2}, \ldots, j_{m}, k-1\right\}, \ldots$, $f\left(j_{m}, \ldots, j_{m}\right) \in\{k-1\}$, but $f(k-1, \ldots, k-1)=k-1$. I.e. $f$ is not $\delta$ function.

Theorem 5. Monotone algebras are not essentially maximal.

Proof. These algebras are not $\delta$ algebras. They are not constant algebras since any constant algebra is not monotone.

\subsection{Linear Algebra}

There are 2 definitions of linear (more precisely, quasilinear) algebras: the definition of Rosenberg [22] and the definition of Yablonski [13].

According to Rosenberg, the quasilinear function

$$
\begin{gathered}
a_{0,0}+a_{1,0} x_{1}+\cdots+a_{1, m-1} x_{1}^{p^{m-1}}+\cdots \\
\ldots+a_{n, 0} x_{n}+\ldots+a_{n, m-1} x_{n}^{p^{m-1}}
\end{gathered}
$$

generates only quasilinear functions for $k=p^{m}$. 
Theorem 6. The quasilinear Rosenberg algebra does not exist.

Proof. It is enough to prove that for any $p$ and $m$ there exists a quasilinear function generating a non-quasilinear function.

Let $k=p^{m+1}$, and let $f$ be $x_{1}^{p^{m}}+x_{2}^{p^{m}}$. This function is quasilinear for $m>0$.

The result of substituting $f$ instead of the first variable in $f$

$$
f_{0}\left(x_{1}, x_{2}, x_{3}\right)=f\left(f\left(x_{1}, x_{2}\right), x_{3}\right)=\left(x_{1}^{p^{m}}+x_{2}^{p^{m}}\right)^{p^{m}}+x_{3}^{p^{m}}
$$

must also be a quasilinear function:

$$
\begin{gathered}
f_{0}\left(x_{1}, x_{2}, x_{3}\right)=a_{0}+a_{10} x_{1}+. .+a_{1 m} x_{1}^{p^{m}} \\
+a_{20} x_{2}+\ldots+a_{2 m} x_{2}^{p^{m}} \\
+a_{30} x_{3}+\ldots+a_{3 m} x_{3}^{p^{m}}
\end{gathered}
$$

Then:

$$
\begin{gathered}
f_{0}(0,0,0)=a_{0}=0, f_{0}(1,0,0)=a_{10}+\ldots+a_{1 m} \\
=1, f_{0}(0,1,0)=a_{20}+\ldots+a_{2 m}=1, \\
f_{0}(1,0,0)+f_{0}(0,1,0)=a_{10}+\ldots+a_{1 m}+a_{20}+\ldots+a_{2 m}=2, \\
f_{0}(1,1,0)=a_{10}+\ldots+a_{1 m}+a_{20}+\ldots+a_{2 m}=2^{p^{m}}
\end{gathered}
$$

i.e. $2 \equiv 2^{p^{m}}$ modulo $p^{m+1}$. Therefore, $p^{m+1}$ divides $2^{p^{m}}-2$. But this is impossible, because $2^{p^{m}}-2$ is odd after dividing by 2 , and $p^{m+1}$ is even after dividing by 2 . Even number cannot divide odd number.

Further definition of Yablonski is used. By this definition, a function is linear if it is linear for usual sum and product operations, and if it is linear for sum and product operations that are dual (with respect to any functions of $s$ ) to ordinary operations.

In follows, a function and algebra are called quasilinear if they are linear for dual operations of sum and product, but not equal to linear for usual operations of sum and product.

For $k=3$, quasilinear algebras do not exist. For $k>3$, quasilinear algebras exist, but they are all dual to linear algebra. Therefore, it suffices to restrict to linear algebras.

Lemma 3 . If $k$ is a prime number, then only $f(x)=a_{0}+$ $x$ is a linear $\delta$ function for $a_{0} \neq 0$.

Proof. Let $f_{0}\left(x_{1}, \ldots, x_{n}\right)=a_{0}+a_{1} x_{1}+\ldots+a_{n} x_{n}$ be a linear $\delta$ function. Then $f_{0}(x, \ldots, x)=a_{0}+a x \neq x$, where $a$ is the sum of all $a_{i>0}$. But if $a \neq 1$, then there exists $x$ such that $a_{0}+a x=x$ (modulo $k$ ), i.e. $f_{0}$ is not a $\delta$ function. Indeed, the function $a_{0}+(a-1) x$ runs through all values, in particular, the value 0 , since $k$ is a prime number. Therefore, $f_{0}(x, \ldots, x)=x$ for $a \neq 1$, and $f_{0}(x, \ldots, x) \neq x$ for $a=1, a_{0} \neq 0$.

Theorem 7. Linear algebras are not essentially maximal.

Proof. Linear algebras belonging to the family $\delta$ are algebras of one-place functions, since they are generated by one-place functions $f(x)=a_{0}+x$. But the algebras of one-place functions are contained in the Slupetski algebra.

\subsection{Cellular Algebras}

The third set of maximal algebras, that are not essentially maximal, is a set of cellular algebras.

Definition 1. (A. I. Maltsev) Cell is an algebra containing all multi-place functions that take less than $h \leq k$ values. Cellular is an algebra containing a cell and a subalgebra of the algebra of one-place functions, in particular, of all one-place functions.

A special case of cellular algebras is the Slupetsky algebra. This algebra contains all multi-place functions that take less than $k$ values and contains the algebra of all one-place functions.

Maximal cellular algebras are fictitious.

Theorem 8. Maximal cellular algebras are not essentially maximal.

Proof. Cellular algebras with $h<k$ are contained in cellular algebras with $h=k$. Cellular algebras with $h=k$ are unions of cellular algebras with $h=k-1$, i.e. they are fictitious. But fictitious algebras are not essentially maximal.

Cellular algebras with $h=k-1$ are not unions of algebras contained in them. However, they are not essentially maximal.

Theorem 9. Cellular algebras with $h=k-1$ are not essentially maximal.

Proof. Cellular algebras with $h=k-1$ are contained in algebras of the type $T_{\mathcal{E}_{i_{1}, \ldots, i_{k-2}}^{k}}^{k}$ [13]. The functions of these algebras take the values $i_{1}, \ldots, i_{k-2}$ for values of variables $i_{1}, \ldots, i_{k-2}$. I.e. for number of values $k-2$. Among the functions with these values, all functions of cellular algebras with $h=k-1$ are present.

\subsection{Algebras of Functions Preserving h-Universal Relations}

These algebras belong to the sixth Rosenberg set. For $k=3$ there should be only one algebra of this family - the Slupetsky algebra. Below it will be proved that for $k=3$ only one algebra of this family exists and it not the Slupetsky algebra. This is another mistake by I. G. Rosenberg.

Definition 2. (I. G. Rosenberg) The algebra of functions that preserve the $h$-universal relation $r\left(x_{1}, \ldots x_{h}\right)$ is determined by the conditions:

a) $h \geq 3$;

b) $\exists m m \geq 1 \wedge h^{m} \leq k$;

c) there is $m$ equivalence relations $r_{i}\left(x_{1} x_{2}\right)$, each of which has $h$ equivalence classes: $X_{i}\{1, \ldots, m\}, j\{1, \ldots, h\}$;

d) all $m h$ equivalence classes do not intersect;

e) relation $r\left(x_{1}, \ldots, x_{h}\right)$ has in each row a pair of variables such that the pair belongs to one of the classes $X_{\mathrm{i}, \mathrm{j}}{ }^{3}$.

All rows of $r\left(x_{1}, \ldots, x_{h}\right)$ have not all values. But function preserving $r$ can have all values. For example, let function $f$ be $f\left(0, x_{2}, \ldots, x_{n}\right)=1, f\left(1, x_{2} \ldots, x_{n}\right)=0, f\left(x_{1}>1, x_{2}, \ldots, x_{n}\right)=x_{1}$. This function has all values, and values of $f$ are values of the first variable. If $x_{l}$ has not all values, then $f$ has not all values, too. Indeed, if $x_{l}$ has not value 0 or 1 , then $f$ has not value 1 or 0 . If

\footnotetext{
${ }^{3}$ I.G. Rosenberg ([22], p.256) formulated it as "at least two of $x_{1}, \ldots, x_{h}$ are equivalent in $r_{i}(i=1, \ldots, m)$ ". D. Lau ([23], p.178) gave another formulation: “ $\left(a_{1}, \ldots, a_{h}\right) \in r \leftrightarrows \forall 1 \leq i \leq m: \exists\{r, s\} \subset\{1, \ldots, h\}: r \neq s \wedge\left(a_{r}, a_{s}\right) \in r_{i}$ ". This formulation is wrong.
} 
$x_{l}$ has not value $\mathrm{j}>1$, then $f$ has not this value, too.

This means that $r$ does not generate Slupecky algebra. This algebra is maximal, but it is absent in Rosenberg's sets. I.G. Roseberg is wrong one more.

The remaining 3 Rosenberg's sets contain essentially maximal algebras.

\subsection{Essentially Maximal Algebras of Functions with $k=3$ and $n=2$}

Further, the results of calculations on a computer are given. These calculations illustrate the structure of essentially maximal algebras.

Numerical calculations are possible with $k=3$ and $n=2$, since the number of all functions is 19683 in this case. With $k=3$ and $n=3$ the number of functions is more than $7 \cdot 10^{12}$. This cannot be calculated. For $k=4$ and $n=2$, the number of functions is more than $4 \cdot 10^{9}$. This can be calculated, but it takes a lot of time.

The restriction in two-place functions is not essential, since two-place functions are generators of many essential algebras. In particular, the generators of algebra $\mathrm{P}_{\mathrm{k}}$ are double functions, and the number of these generators is very large. For $k=3$, this number is 3774 [24], and for $k=4$, this number is 942 897552 [25].

The largest number of essentially maximal algebras belongs to algebras that preserve central relations. The number of these algebras is 9 . These are algebras of functions with one center ( 3 constant algebras), with two centers ( 3 algebras) and with three centers (also 3 algebras, but only 1 is essentially maximal). Each of this triple contains dual algebras.

Each algebra with one center contains 6561 two-place functions and does not contain $\delta$ functions.

If an algebra contains functions that preserve relations with two centers, then this algebra contains 3888 two-place functions, of which 648 functions are $\delta$. This algebra is essentially maximal, too.

Algebras are not essentially maximal if they contain all functions that preserve relations with three centers. Each such algebra is a union of three algebras. Two of these algebras contain 512 two-place functions and no $\delta$ function. These algebras are not essentially maximal. The third algebra contains 849 two-place functions, of which $256 \delta$ functions are. This algebra is not maximal, since it is contained in maximal algebra, but it is essentially maximal.

There are 3 algebras of functions that preserve non-trivial equivalence relations. These algebras are dual. These algebras are not essentially maximal. Each algebra is the union of four algebras.

The first algebra contains 16 functions and does not contain $\delta$ functions. This algebra is not essentially maximal.

The second algebra contains 525 functions, of which $150 \delta$ functions are. This algebra is essentially maximal.

The third algebra contains 672 two-place functions, of which $136 \delta$ functions are. This algebra is not essentially maximal; it is contained in the algebra of functions that preserve relations with two centers.

The fourth algebra contains 1152 two-place functions and $192 \delta$ functions. This algebra is not essentially maximal. It is also contained in the algebra of functions that preserve relations with 111111 two centers.

There remains a maximal algebra of self-dual functions. This algebra contains 27 double functions and $18 \delta$ functions. This algebra is essentially maximal.

\section{Conclusion}

A classification of $k$-valued functions is constructed for any natural value of $k$. The top level is a class containing all the functions of value $k$. At the next level, there are classes that differ in the number of functions in the minimal basis of their algebras. A special class contains all algebras generated by single bases. Unlike other bases, single bases do not intersect. These bases are foundation for constructing a natural classification of functions, i.e. classifications with disjoint subclasses. In such a classification, each function belongs to a single subclass.

Each algebra in a class of algebras with single bases has a set of bases, and this set is a class of functions in the natural classification. Each function belongs to the only such class, since each function generates a unique algebra.

Therefore, all algebras from classes of algebras with non-one-member bases are fictitious, since they are useless for the natural classification of functions.

The theory of algebras of functions has the continual set of fictitious algebras. But the set of essential algebras is countable. Therefore, the statement about the fundamental difference between the algebra of two-valued functions and algebras of many-valued functions turned out to be erroneous.

The next level of classification of functions was constructed. This level contains all classes that contain essentially maximal algebras. But the families of monotone algebras, linear algebras, and cellular algebras do not contain essentially maximal algebras. The maximal algebras of families of self-dual algebras, algebras of functions that preserve nontrivial equivalence relations, and algebras of functions that preserve relations with one and two centers are essentially maximal. As a rule, maximal algebras preserving relations with three or more centers are not essentially maximal, but each algebra contains one subalgebra, which is essentially maximal.

Thus, the number of essentially maximal algebras is equal to the number of maximal algebras in these three sets of algebras.

A powerful tool for constructing a classification of algebras of functions is a family of functions that preserve some constants. It is proved that the set of bases of subalgebras is the set of functions of the same family, which simplified the construction of essentially maximal subalgebras. The algebras of functions, that preserve one constant or preserve 0 constants, turned out to be essentially maximal.

It is proved that there is no algebra of Rosenberg quasilinear functions. The set of algebras of functions, that preserve $h$-universal relations, is also erroneous. This set is replaced by a family of cellular algebras. 


\section{References}

[1] E. L. Post, Introduction to a general theory of elementary propositions Amer. J. Math., (43), 163-185 (1921).

[2] E. L. Post, Two-valued iterative systems of mathematical logic, Princeton, Princeton Univ. Press (1941).

[3] A. V. Kuznetsov, On means for detecting of non-deductibility and inexpressibleness (Russian), in Logical conclusion, M., Science, 5-33 (1979).

[4] S. S. Marchenkov, On Expressibility of Multivalued Logic Functions in some Logic-Functional Languages (Russian), Discrete Mathematics, (11:4), 110-126 (1999).

[5] S. S. Marchenkov, S-classification of many-valued logic functions, Discrete Mathematics (9:3), 125-152 (1997).

[6] S. S. Marchenkov, S-classification of many-valued logic functions (Russian), M., Physmatlit (2001).

[7] S. S. Marchenkov, Equational closure (Russian), Discrete Mathematics, (17:2), 117-126 (2005).

[8] S. S. Marchenkov, On structure of equationally closed classes (Russian), Discrete Mathematics, (18), 18-30 (2006).

[9] S. S. Marchenkov, Definability in language of functional equations of countably-valued logic (Russian), Discrete Mathematics, (25:4), 13-23 (2013).

[10] S. S. Marchenkov, On FE-Precomplete Classes of Countable Logic (Russian), Discrete Mathematics, (28:2), 51-57 (2016).

[11] A. V. Kuznetsov, Algebra of logic and its generalization (Russian), in Mathematics in USSR for 40 years, M. Physmatlit, 102-115 (1959).

[12] E. Yu. Zakharova, V. B. Kudryavtsev, S. V. Yablonsky, On precomplete classes in k-valued logics (Russian), Reports of USSR Academy of Sciences (186:3), 509-513 (1969).

[13] S. V. Yablonsky, Functional constructions in k-valued logic (Russian), Proceedings of Mat. Institute of the USSR Academy of Sciences. V. A. Steklova, (51) 5-142 (1958).

[14] D. Lau, Submaximale klassen von $P_{3}$, J. Inf. Process. Cybern. EIK (18:4/5), 227-243 (1982).

[15] I. G. Rosenberg, The number of maximal closed classes in the set of functions over a finite domain, J. combinat. Theory, Ser. A (14), (1-7) (1973).

[16] Yu. I. Yanov, A. A. Muchnik, On the existence of $k$-valued closed classes that do not have a finite basis (Russian), Doc. USSR Academy of Sciences, (127:1), 44-46 (1959).

[17] M. A. Malkov, Classification of Closed Sets of Functions in Multi-valued Logic, SOP Transactions on applied Math., (1:3), 96-105 (2014).

[18] M. A. Malkov Classifications of Boolean Functions and Their Closed Sets, SOP Transactions on applied Math., (1:2), 172193 (2014).

[19] A. I. Maltsev, Iterative Post Algebras, Novosibirsk, Novosib. state un-t (1976).

[20] M. A. Malkov, Finite Closed Sets of Functions in Multi-Valued Logic, Pure and Appl. Math J., (6:1), 14-24 (2017).

[21] S. V. Yablonsky, G. I. Gavrilov, V. B. Kudryavtsev, Functions of the algebra of logic and Post classes, M., Science (1966).

[22] I. G. Rosenberg, Complete sets for finite algebras Math. Nachr., (4), 253-258 (1970).

[23] D. Lau, Function algebras on finite sets, Berlin, Heidelberg, New York, Springer monographs in mathematics (2006).

[24] N. M. Martin, The Sheffer functions of tree valued logic, $J$. Symb. $\log (19), 45-51$ (1954).

[25] M. A. Malkov Complete generators in 4-valued logic and Rousseau's results, Intern. J. of Math. and its Applications, (2:6), 49-57 (2014). 